\title{
Evaluación ecotoxicológica por disponibilidad a metales pesados durante periodo de lluvia en el ecosistema San Juan, Santiago de Cuba - Cuba
}

Ecotoxicological evaluation for availability to heavy metals during period of rain San Juan ecosystem, Santiago Cuba - Cuba

Recibido: enero de 2016 | Revisado: marzo de 2016 | Aceptado: abril de 2016

George Argota Pérez ${ }^{\text {I }}$

1 Centro de Investigaciones Avanzadas y Formación Superior en Educación, Salud y Medio Ambiente "AMTAWI". Departamento de Puno, Perú.

george.argota@gmail.com

\begin{abstract}
Pollution of aquatic ecosystems by heavy metals is a major environmental problem solving, because their analysis and interpretation on the relevance has finally resorting to biological matrices developed in natural environments. The aim of this research was to assess the ecotoxicology by exposure to heavy metals in the ecosystem San Juan Santiago de Cuba - Cuba. To do an investigation which lasted from May to November 2015, understanding this time the rainy period was performed. Abiotic sampling water matrix and the species was Gambusia punctata random probability, while quantitative technique used corresponded to structured observation. Continuous quantitative variables corresponded to dissolved oxygen, $\mathrm{pH}$, electrical conductivity total solids, total alkalinity, total hardness and who were determined in- situ. In the case of chemical and biochemical oxygen demands they were analyzed under laboratory conditions. Concentrations of $\mathrm{Cu}, \mathrm{Zn}, \mathrm{Pb}$ and $\mathrm{Cd}$ were determined in water and bioaccumulate in gills, liver and brain BioMonitor. Wet elements quantitated by atomic absorption spectrometry by inductively coupled plasma axial view. It was noted that in general physicochemical parameters were found outside the allowable value by the regulatory standard used and in the case of the elements showed bioavailable concentrations, then finding bioaccumulate in target organs.

Among the stations no statistically significant differences $(p \leq 0.05)$ and can be interpreted as persistence and mobility conditions were observed in similar form. It was concluded that the waters of the San Juan ecosystem showed physicochemical to the availability of metals, conditions can be corroborated by bioaccumulation in the tissues analyzed, because there was ecotoxicological risk for the species that develops in a natural environment.
\end{abstract}

Keywords: biomarkers , ecotoxicology, San Juan ecosystem, Gambusia punctata; chemical speciation, heavy metals, Santiago de Cuba - Cuba

\section{Resumen}

La contaminación de los ecosistemas acuáticos por metales pesados es uno de los principales problemas ambientales por resolver, por cuanto su análisis e interpretación es sobre la relevancia en recurrir finalmente, a las matrices biológicas desarrolladas en ambientes naturales. El objetivo 
de la presente investigación fue evaluar la ecotoxicología por exposición a metales pesados en el ecosistema San Juan de Santiago de Cuba-Cuba. Para ello se realizó una investigación que transcurrió desde mayo hasta noviembre del 2015, comprendiendo esta época al periodo de lluvia. El muestreo sobre la matriz abiótica agua, así como en la especie Gambusia punctata fue probabilístico aleatorio, mientras que la técnica cuantitativa empleada correspondió a la observación estructurada. Las variables cuantitativas continuas correspondieron al oxígeno disuelto, $\mathrm{pH}$, conductividad eléctrica, alcalinidad total, dureza total y sólidos totales, que se determinaron insitu. En el caso de las demandas química y bioquímica de oxígeno, se analizaron en condiciones de laboratorio. Se determinaron concentraciones de $\mathrm{Cu}, \mathrm{Zn}, \mathrm{Pb}$ y $\mathrm{Cd}$ en las aguas como bioacumulados en branquias, hígado y cerebro del biomonitor. Los elementos se cuantificaron vía húmeda mediante espectrometría de absorción atómica por plasma inductivamente acoplado con vista axial. Se observó que los parámetros físico-químicos en general, se encontraron fuera del valor permisible por la norma reguladora utilizada y en el caso de los elementos, mostraron concentraciones biodisponibles, encontrándose luego bioacumulados en los órganos dianas. Entre las estaciones no se observaron diferencias estadísticamente significativas $(p \leq 0.05)$, pudiendo interpretarse como persistencia y movilidad de las condiciones en forma similares. Se concluyó que las aguas del ecosistema San Juan, mostraron condiciones físico-químicas para la disponibilidad de metales, pudiendo ser corroboradas mediante la bioacumulación en los tejidos analizados, por cuanto existió riesgo ecotoxicológico para la especie que se desarrolla en un ambiente natural.

Palabras claves: biomarcadores, ecotoxicología, ecosistema San Juan; Gambusia punctata; metales pesados; Santiago de Cuba-Cuba.

\section{Introducción}

Conforme a la definición de Paustenbach (1990, p.), "riesgo es la probabilidad de que ocurra un efecto adverso a nivel individual o poblacional, por la exposición a una concentración o dosis específica de un agente peligroso". Desde luego, esto abarca dos dimensiones: a) la posibilidad de que haya un resultado negativo; y b) la incertidumbre sobre la aparición, duración y magnitud del resultado adverso (Albert, 1997). Por lo que riesgo también se puede definir como: situación o acción en donde son posibles dos o más resultados; se desconocen las posibilidades de aparición de un resultado en parti- cular y; al menos una de las posibilidades es indeseable (Silveira y Oliveira-filho, 2013).

Carpenter (1995), señaló que la evaluación de riesgos ambientales (ERA) sigue las reglas de la teoría de la probabilidad mediante una expresión de todos los posibles valores de cada parámetro analizado. En el caso del Management Programme for the Gorgon Development (MPGD, 2004), indicó que la ERA es un proceso que evalúa la probabilidad y consecuencia de un impacto adverso que ocurre como resultado de la exposición a uno o más estresores. Sin embargo, Van der Oost, Beyer y Vermeulen (2003), argumentan que deberían analizarse los biomarcado- 
res como proteínas de estrés, metalotioneínas, parámetros hematológicos, inmunológicos, genotóxicos, endocrinos, etc. así como marcadores de bioacumulación por ejemplo en peces y su empleo en ERA.

Para el caso de uno de los agentes químicos contaminantes como son los metales, la tabla periódica incluye unos 70 elementos metálicos, de ellos 59 pueden ser considerados "metales pesados", siendo solo aquellos con peso atómico mayor al Fe $(55.85 \mathrm{~g} / \mathrm{mol})$. Con esta precisión se excluirían metales con pesos atómicos menores al $\mathrm{Fe}$, que con frecuencia pueden ser metales contaminantes como el V (50.95), Mn (54.44), Cr (52.01) y otros que realmente no son metales como As, F y P. Es por ello, que resulta mejor hablar de contaminación por "elementos traza”, dado que la mayoría de los contaminantes inorgánicos son "metales pesados" (Plant, D. Smith, B. Smith \& Williams, 2001).

Entre las principales fuentes antropogénicas para los elementos traza se encuentran las actividades agrícolas, metalúrgicas, la energía tanto en su producción como transporte, además de los productos micro-electrónicos y la deposición de residuos (Zhou, Zhang, Fu, Shi \& Jiang, 2008).

A nivel de ecosistemas acuáticos, los metales pesados se transforman a través de procesos biogeoquímicos en especies con distintas características físico-químicas (Tercier \& Taillefert, 2008). La medición de los niveles de metales disueltos en las aguas naturales como indicador de matriz ambiental, presenta varios problemas analíticos debido a que, en múltiples ocasiones los metales se encuentran en bajas concentraciones o cercanos a los límites de detección de los métodos empleados, por lo que es necesario realizar preconcentraciones de los mismos. La medición de las concentraciones totales provee de una estimación del grado de contaminación que presentan las aguas, pero la fracción biodisponible de cada especie metálica es la que presenta una mayor relevancia toxicológica para la acumulación por los organismos ( Rainbow, 1995; Páez-Osuna \& Osuna-Martínez, 2011).

Actualmente, la distribución, movilidad, disponibilidad biológica y toxicidad de los elementos químicos no son funciones de la concentración total de metales, sino de la forma química en la que se encuentren. Los elementos forman parte de distintas moléculas en función de su estado de oxidación y del medio en que se encuentren, por lo que el análisis de especiación es una herramienta esencial para la evaluación de riesgos en el medio ambiente, permitiendo que se realicen diagnósticos y controles más efectivos de los elementos trazas. Es por ello que según Sigg et al. (2006), toda legislación ambiental debe estar basada en la concentración máxima permisible de especies químicas, en vez de las concentraciones totales de los elementos.

De conjunto a lo planteado anteriormente, durante los últimos años se ha intensificado la búsqueda de métodos de estudio para detectar los impactos sobre los ecosistemas acuáticos con motivo de poder diseñar e implementar medidas preventivas. En este caso, el uso y desarrollo de biomarcadores han cobrado un interés creciente para evaluar el riesgo de una sustancia o mezcla química potencialmente tóxica, ya que los biomarcadores constituyen valiosos parámetros o indicadores de la presencia de sustancias exógenas o cambios biológicos como respuestas a distintos xenobióticos (West et al., 2006), donde finalmente toda evaluación a realizarse tanto de parámetros físico-químicos, contaminantes de interés y en biomarcadores, podrían analizarse, simultáneamente ,con predicción de riesgo para un determinado ecosistema.

El objetivo de la presente investigación fue evaluar la ecotoxicología por exposición a metales pesados en el ecosistema San Juan de Santiago de Cuba-Cuba. 


\section{Materiales y Métodos}

\section{Objeto de investigación y periodo de es- tudio}

Las aguas del ecosistema San Juan, ubicado en la ciudad de Santiago de Cuba-Cuba, recorren una longitud aproximada de $23.0 \mathrm{Km}^{2}$ (Figura 1), en que el río principal se descargan diversos efluentes con cargas contaminantes de naturaleza orgánica como inorgánica, encontrándose dentro de esta última naturaleza determinadas concen- traciones de metales pesados. Las aguas de este ecosistema, revisten de gran importancia para la ciudad, ya que en esta cuenca hidrográfica del mismo nombre, existen identificados un gran número de pozos que son utilizados para el abastecimiento potable a la población humana.

La evaluación ecotoxicológica por exposición a metales pesados en el ecosistema San Juan se realizó desde mayo hasta noviembre del 2015, correspondiéndose dicha época al periodo de lluvia.

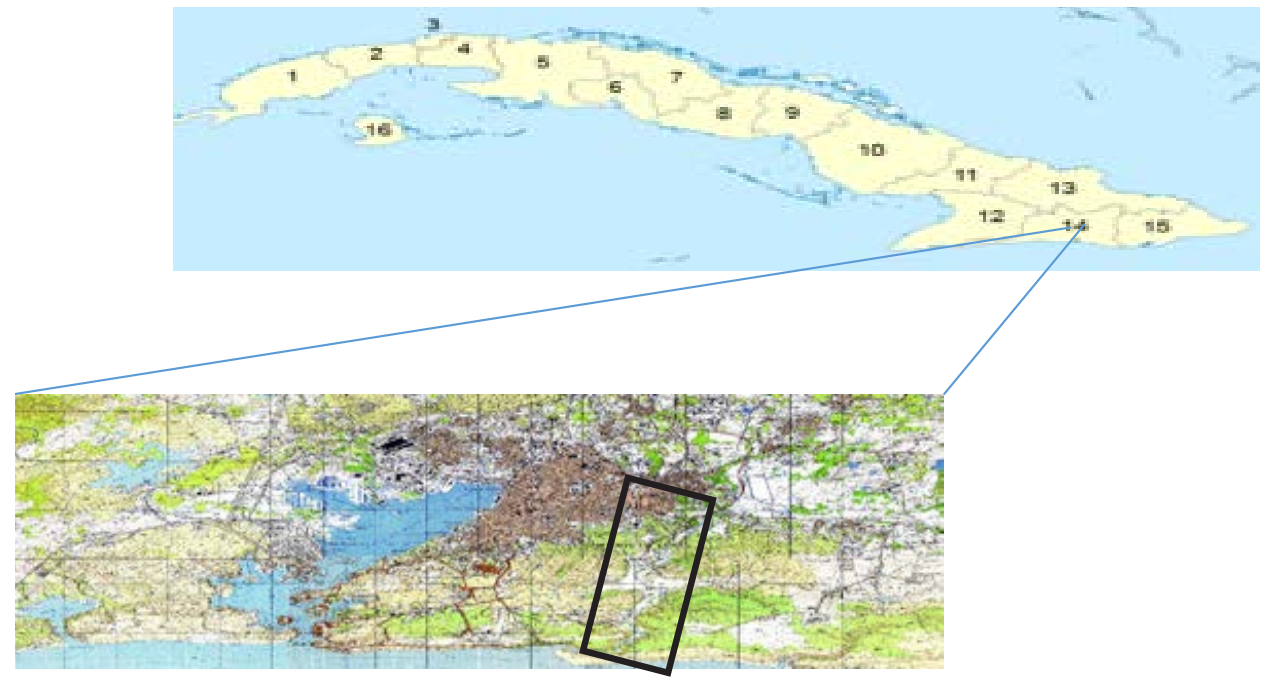

Figura 1. Ecosistema San Juan (rectángulo) en Santiago de Cuba-Cuba. Fuente: Mapa de Cuba (Wikipedia, Historia territorial de Cuba). Consultado 09/10/2015. Disponible en https://es.wikipedia.org/wiki/ Historia_territorial_de_Cuba. Mapa cartográfico. Centro Nacional de Investigaciones Sismológicas-Cuba, 2015.

\section{Población y muestra}

En la presente investigación de tipo explicativa se analizaron en muestras de aguas los parámetros físico-químicos de calidad ambiental correspondiente al oxígeno disuelto, $\mathrm{pH}$, conductividad eléctrica, alcalinidad total, dureza total, sólidos totales, demanda química de oxígeno y demanda bioquímica de oxígeno, además de concentraciones referidas a $\mathrm{Cu}, \mathrm{Zn}, \mathrm{Pb}$ y $\mathrm{Cd}$, que fueron además determinados en las branquias, hígado y cerebro de la especie Gambusia punctata, la cual fue utilizada como organismo natural del ecosistema.
La matriz abiótica agua, así como la captura de los individuos pertenecientes a la especie Gambusia punctata se analizaron por triplicado, a partir de un muestreo probabilístico aleatorio con una frecuencia trimestral de análisis.

Fueron seleccionadas seis estaciones de muestreo, las cuales se escogieron a partir de un muestreo no probabilístico por conveniencia, teniendo como principal criterio de selección su estado de convergencia tributaria o zona de mezcla, donde se requirió de condiciones prácticas de accesibilidad al lugar, posibilidad de muestreo en ambas orillas 
del río, profundidad, existencia de construcciones cercanas o accidentes geográficos del relieve muy llamativos que pudieran servir como puntos de referencia.

\section{Análisis de parámetros físico-químicos de calidad ambiental y metales pesados en aguas}

El muestreo de las aguas se realizó utilizando una vara plástica de 1.5 metro de largo con un vaso de precipitado plástico de $1 \mathrm{~L}$ de capacidad. Las muestras se tomaron con cuidado de la capa superficial sin remover el sedimento, fundamentalmente, en lugares poco profundos. El volumen de muestra tomada en cada estación fue aproximadamente de 5 L según (ISO 1980, 1991, 1994). Luego, las muestras se homogenizaron en tanque de polietileno previamente endulzado con las aguas del propio ecosistema.

Los parámetros físico-químicos de calidad ambiental de las aguas que se analizaron fueron oxígeno disuelto (OD: $\left.\mathrm{mg} / \mathrm{L}^{-1}\right), \mathrm{pH}$, conductividad eléctrica (CE: $\left.\mu_{s} / \mathrm{cm}^{-1}\right)$, alcalinidad total (AT: $\mathrm{mg} / \mathrm{L}^{-1}$ ), dureza total (DT: $\left.\mathrm{mg} / \mathrm{L}^{-1}\right)$, sólidos totales (ST: $\left.\mathrm{mg} / \mathrm{L}^{-1}\right)$, demanda bioquímica de oxígeno $\left(\mathrm{DBO}_{5,20}: \mathrm{mg} / \mathrm{L}^{-}\right.$ $\left.{ }^{1}\right)$ y demanda química de oxígeno (DQO: $\left.\mathrm{mg} / \mathrm{L}^{-1}\right)$.

El OD, pH, CE, AT, DT y los ST fue medido in-situ, mediante el analizador multiparamétrico con marca HI 98.28 (HANNA).
La $\mathrm{DBO}_{5,20}$ fue determinada por el método del respirómetro de Warburg, mientras que la DQO fue determinada, según el método del dicromato empleándose Kits provenientes de HYDROCHEC/WPA.

El OD, pH, $\mathrm{DBO}_{5,20}$ y DQO utilizaron para su evaluación la Norma Cubana: NC. 1999 - Evaluación de los Objetos Hídricos de Uso Pesquero.

En el caso de la CE, AT, DT y ST se evaluaron de acuerdo con lo establecido por la Norma Cubana: NC. 1985 - Higiene comunal. Agua potable. Requisitos sanitarios y muestreos.

La curva de calibración se realizó a partir de reactivos de calidad espectral, verificando las características metrológicas del método, usando valores certificados de muestras patrones de referencia de cada tipo de muestra.

Se comenzó a partir de una solución patrón de $1000 \mathrm{mg} / \mathrm{L}^{-1}$ y los estándares de calibración se prepararon por dilución de la misma con solución de $\mathrm{HNO}_{3}(0.7 \mathrm{M})$.

Se determinaron los contenidos de $\mathrm{Cu}$, $\mathrm{Zn}, \mathrm{Pb}$ y $\mathrm{Cd}$ mediante mediciones con el Espectrómetro de Absorción Atómica por Plasma Inductivamente Acoplado con Vista Axial (ICP-AES) de la firma alemana Spectro-Arco, según las condiciones reportadas en la Tabla 1. Todos los análisis se realizaron por triplicado a partir de muestras independientes.

Tabla 1

\section{Condiciones instrumentales}

\begin{tabular}{l|c}
\hline \multicolumn{1}{c|}{ PARÁMETROS } & CONDICIONES \\
\hline Tipo de nebulizador & Meinhard (concéntrico) \\
Gratícula & 2400 líneas $/ \mathrm{mm}$ \\
Frecuencia & $27.0 \mathrm{MHz}$ \\
Energía del plasma & $1.4 \mathrm{KW}$ \\
Velocidad de flujo del gas & $18.8 \mathrm{~L} / \mathrm{min}$ \\
Pre flujo & $2.0 \mathrm{~mL} / \mathrm{min}$ \\
Velocidad de bombeo de la muestra & $15.0 \mathrm{r} . \mathrm{p} . \mathrm{m}$. \\
Tiempo de integración & $3.0 \mathrm{segundos}$ \\
Ranura de entrada de la radiación & $25.0 \mu \mathrm{m}$ \\
\hline
\end{tabular}


Análisis de la bioacumulación de $\mathrm{Cu}, \mathrm{Zn}$, $\mathrm{Pb}$ y $\mathrm{Cd}$ en las branquias, hígado y cerebro del biomonitor Gambusia punctata

Se seleccionaron peces adultos de la especie Gambusia punctata, que se clasificaron por sexo longitud $(2.1-3.0 \mathrm{~cm}$ y $3.1-4.0 \mathrm{~cm})$ y órgano en la bioacumulación (branquias, hígado y cerebro).

Los individuos, una vez muestreados fueron colocados en posición ventral y mediante la utilización de una pinza de sujeción dentada, se realizó una conización posterior a los orificios nasales correspondiente en la región cefálica hasta visualizar y extraer con ayuda de una pinza de disección lisa el cerebro.

Posteriormente, la especie fue colocada en posición dorsal y mediante la utilización de dos pinzas de disección lisas, los opérculos para extraer los arcos branquiales. En esta misma posición, se realizó un corte con una tijera curva para extraer el hígado. se abrieron.

Los órganos una vez extraídos fueron tratados individualmente en forma de pool y colocados en estufa a $70^{\circ} \mathrm{C}$ durante 48 horas para su secado total (Argota et al., 2013). Las muestras de cada órgano fueron trituradas y homogenizadas utilizando un mortero de ágata (U.S. Enviromental Protection Agency [USEPA], 2000).

Para el análisis se pesó en balanza analítica $0.5 \mathrm{~g}$ de las muestras, colocándose las mismas en vasos de precipitados de 250.0 $\mathrm{mL}$. Se adicionaron $5.0 \mathrm{~mL}$ de una mezcla de ácidos $\mathrm{HClO}_{4}: \mathrm{H}_{2} \mathrm{SO}_{4}(7: 1)$ y $15.0 \mathrm{~mL}$ de $\mathrm{HNO}_{3}$ concentrado y la digestión se efectuó en una plancha de calentamiento a $80^{\circ} \mathrm{C}$, hasta la evaporación total de la mezcla de ácidos. Se añadió nuevamente $5.0 \mathrm{~mL}$ de $\mathrm{HNO}_{3}$ concentrado y se calentó hasta la aparición de sales húmedas. Finalmente, se trasvasó cuantitativamente a frasco volumétrico de $25.0 \mathrm{~mL}$ con la ayuda de una disolución de ácido nítrico $0.7 \mathrm{M}$.
La cuantificación se realizó igualmente con el Espectrómetro de Absorción Atómica por Plasma Inductivamente Acoplado con Vista Axial (ICP-AES) de la firma alemana Spectro-Arco, según las condiciones reportadas en la Tabla 1.

\section{Análisis estadístico}

Para el tratamiento de los resultados se aplicaron métodos estadísticos que correspondieron al análisis de la varianza factorial con réplicas para definir las fuentes de variación significativas y la prueba de intervalos múltiples de Duncan para determinar las magnitudes individuales de las diferencias que resulten significativas según lo expresado por Montgomery (1991).

Todos los cálculos se realizaron utilizando el software profesional Statgraphics (Statpoint Technologies, 1994-2001), donde los resultados se consideraron significativos a un nivel de confianza del 95\% ( $\mathrm{p} \leq 0.05)$.

\section{Bioética en la investigación}

La eutanasia para la especie, consistió en preparar bolsas esterilizadas con hielo que se depositaron en diferentes recipientes de cristal con agua tratada, procurando que durante la inmersión de los ejemplares, fueran tranquilizados por hipotermia inmediata, inhibiéndose toda función biológica que permita realizar finalmente la disección.

\section{Resultados}

Evaluación de parámetros físico-químicos de calidad ambiental y metales pesados en aguas

En la Tabla 2, se muestran los resultados obtenidos de los parámetros físico-químicos por estaciones de muestreo, su análisis de componentes principales, el peso (Tabla $3 \mathrm{y}$ 4) y representación gráfica de dichos parámetros físico-químicos según el propio análisis de componentes principales (Figura 2). 
Tabla 2

Parámetros físico - quimicos por estaciones de muestreo

\begin{tabular}{|c|c|c|c|c|c|c|c|c|c|}
\hline \multirow{2}{*}{ Estación } & \multirow{2}{*}{ Período } & \multicolumn{8}{|c|}{ Parámetros físico - químicos } \\
\hline & & OD & $\mathrm{pH}$ & $\mathrm{CE}$ & AT & DT & ST & $\mathrm{DBO}_{5,20}$ & DQO \\
\hline 1 & \multirow{6}{*}{ Lluvia } & 5.1 & 7.2 & 132.2 & 160 & 220 & 543.33 & 22 & 112 \\
\hline 2 & & 3.5 & 6.8 & 122.3 & 212 & 200 & 553.33 & 11.2 & 132 \\
\hline 3 & & 4.7 & 7.3 & 140.8 & 228 & 272 & 473.33 & 5.59 & 247 \\
\hline 4 & & 3.0 & 6.8 & 138.9 & 220 & 300 & 560 & 39 & 312 \\
\hline 5 & & 3.1 & 6.7 & 122.7 & 212 & 312 & 543.33 & 16 & 332 \\
\hline 6 & & 3.2 & 6.7 & 150.5 & 220 & 340 & 590 & 15 & 432 \\
\hline \multicolumn{2}{|c|}{ Referencia } & 5.0 & $\begin{array}{c}6.5- \\
8.5\end{array}$ & $\begin{array}{c}\leq \\
100.0\end{array}$ & $\begin{array}{c}\leq \\
200.0\end{array}$ & $\begin{array}{c}\leq \\
200.0\end{array}$ & $\leq 500.0$ & 3.0 & $\leq 15.0$ \\
\hline
\end{tabular}

Como parámetros de calidad del agua se entienden aquellos propios de la composición de los ríos, así quedan englobados dentro de éstos los parámetros físico-químicos siempre que se encuentren dentro de los rangos propios de las aguas superficiales libre de la influencia del hombre, el cual hace sobrepasar los valores de estos parámetros a niveles muy superiores a los esperados. La determinación de los parámetros físico-químicos se realiza en general, para tener un criterio evaluativo de la calidad de los cuerpos de agua, independientemente del tipo de uso.

\section{Tabla 3}

Análisis de componentes principales dado parámetros fisico-quimicos

\begin{tabular}{cccc}
\hline Parámetros & eigenvalor & $\begin{array}{c}\text { porcentaje de } \\
\text { varianza }\end{array}$ & $\begin{array}{c}\text { porcentaje } \\
\text { acumulado }\end{array}$ \\
\hline DBO & 3.82751 & 47.844 & 47.844 \\
DQO & 2.09495 & 26.187 & 74.031 \\
OD & 1.19096 & 14.887 & $\mathbf{8 8 . 9 1 8}$ \\
pH & 0.756032 & 9.450 & 98.368 \\
ST & 0.130545 & 1.632 & 100.000 \\
CE & $2.75923 \mathrm{E}-16$ & 0.000 & 100.000 \\
AT & $6.4747 \mathrm{E}-17$ & 0.000 & 100.000 \\
DT & 0.0 & 0.000 & 100.000 \\
\hline
\end{tabular}

Tabla 4

Tabla de pesos de los componentes dado parámetros físico-químicos

\begin{tabular}{ccc}
\hline Parámetro & $\begin{array}{c}\text { Componente } \\
1\end{array}$ & $\begin{array}{c}\text { Componente } \\
2\end{array}$ \\
\hline DBO & 0.225364 & -0.277342 \\
DQO & 0.419003 & 0.244505 \\
OD & -0.463647 & 0.194736 \\
PH & -0.458206 & 0.0998363 \\
ST & 0.370447 & -0.438302 \\
CE & -0.0628343 & -0.482711 \\
AT & 0.23611 & 0.578995 \\
DT & 0.389615 & 0.234639 \\
\hline
\end{tabular}

Dado los valores de la Tabla 4, donde se han estandarizado restándoles su media y dividiéndolos entre sus desviaciones estándar, quedaría, por ejemplo, para el primer componente principal, la siguiente ecuación:

$$
\begin{gathered}
0.225364^{*} \mathrm{DBO}+0.419003^{*} \mathrm{DQO} \\
-0.463647^{*} \mathrm{OD}-0.458206^{*} \mathrm{pH}+ \\
0.370447^{*} \mathrm{ST}-0.0628343^{*} \mathrm{CE}+ \\
0.23611^{*} \mathrm{AT}+0.389615^{*} \mathrm{DT}
\end{gathered}
$$




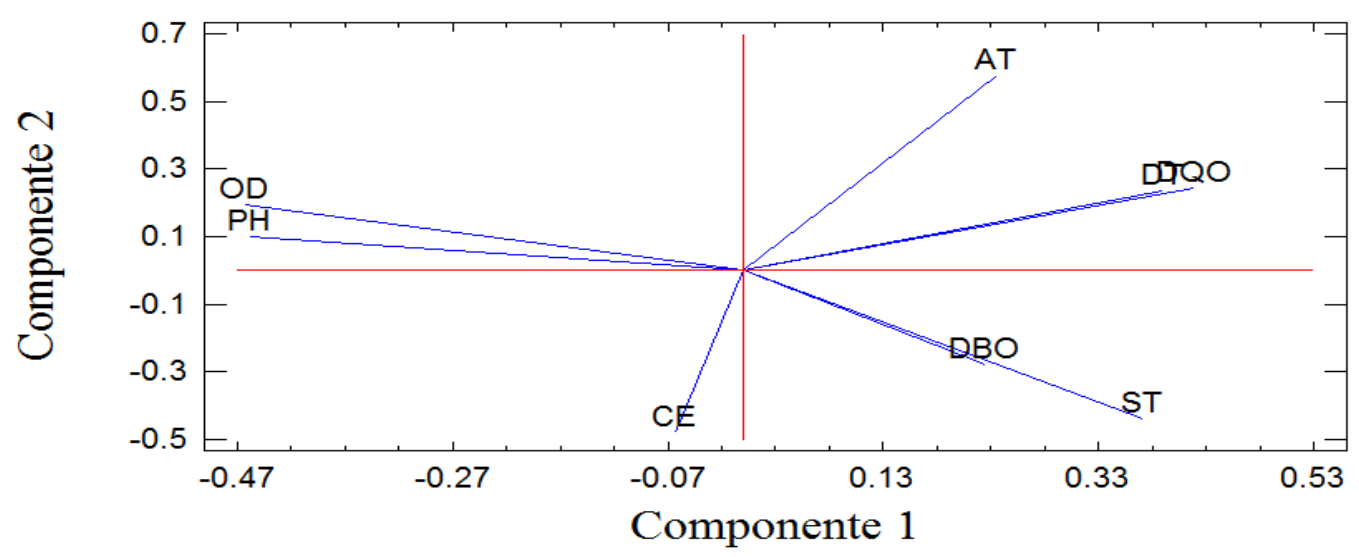

Figura 2. Componentes principales en 2D de parámetros físico-químicos.

En la Tabla 5, se muestra las concentraciones de la disponibilidad de metales pesados en agua, donde no se observó di- ferencias estadísticas significativas entre las estaciones (Tabla 6).

Tabla 5

Concentración de metales pesados en agua (ppm)

\begin{tabular}{ccccc}
\hline Estación & $\mathbf{C u}$ & $\mathbf{Z n}$ & $\mathbf{P b}$ & $\mathbf{C d}$ \\
\hline 1 & $9.21 \pm 1.23$ & $20.2 \pm 3.45$ & $0.11 \pm 0.07$ & $0.008 \pm 0.001$ \\
2 & $14.2 \pm 1.03$ & $28.1 \pm 2.21$ & $0.17 \pm 0.06$ & $0.13 \pm 0.002$ \\
3 & $9.24 \pm 1.23$ & $18.5 \pm 2.59$ & $0.12 \pm 0.03$ & $0.007 \pm 0.001$ \\
4 & $15.2 \pm 2.26$ & $27.2 \pm 2.17$ & $0.16 \pm 0.05$ & $0.12 \pm 0.02$ \\
5 & $7.76 \pm 0.74$ & $21.6 \pm 2.30$ & $0.11 \pm 0.04$ & $0.008 \pm 0.002$ \\
6 & $14.3 \pm 1.68$ & $30.3 \pm 2.33$ & $0.16 \pm 0.03$ & $0.13 \pm 0.02$ \\
\hline
\end{tabular}

La Tabla ANOVA descompone la varianza de los datos en dos componentes: un componente entre grupos y un componente dentro-de-grupos. La razón-F, que en este caso es igual a 0.880107 , es el cociente entre el estimado entre-grupos y el estimado den- tro-de-grupos. Puesto que el valor-P de la razón-F es mayor o igual que 0.05 , no existe una diferencia, estadísticamente, significativa entre las medias de las seis estaciones con un nivel del $95.0 \%$ de confianza.

Tabla 6

Análisis de la varianza (ANOVA)

\begin{tabular}{cccccc}
\hline Fuente & $\begin{array}{c}\text { Suma de } \\
\text { Cuadrados }\end{array}$ & $\begin{array}{c}\text { Grados de } \\
\text { libertad }\end{array}$ & $\begin{array}{c}\text { Cuadrado } \\
\text { Medio }\end{array}$ & Razón-F & Valor-P \\
\hline Entre grupos & 476.403 & 5 & 95.2806 & 0.88 & $\mathbf{0 . 4 9 6 3}$ \\
Intra grupos & 14939.9 & 138 & 108.26 & & \\
Total (Corr.) & 15416.3 & 143 & & & \\
\hline
\end{tabular}


En la Tabla 7, se muestran las concentraciones promedio de metales en los órganos dianas de la especie biomonitor Gambusia punctata, correspondiendo solo a la estación 1 y 6 respectivamente, donde en las branquias se encontraron las mayores concentra- ciones y en el cerebro las menores, respectivamente. Asimismo, no se encontraron diferencias estadísticamente significativas $(\mathrm{p} \leq 0.05)$ entre los valores calculados como sumatorias en las estaciones.

Tabla 7

Concentración de metales por órganos dianas (ppm)

\begin{tabular}{cccccc}
\hline Estación & Órganos & $\mathbf{C u}$ & $\mathbf{Z n}$ & $\mathbf{P b}$ & $\mathbf{C d}$ \\
\hline \multirow{2}{*}{1} & branquias & $40.2 \pm 2.43$ & $47.9 \pm 2.56$ & $0.022 \pm 0.03$ & $15.8 \pm 1.71$ \\
& hígado & $34.3 \pm 1.39$ & $66.5 \pm 2.17$ & $0.008 \pm 0.02$ & $0.007 \pm 0.03$ \\
& cerebro & $0.016 \pm 0.05$ & $0.020 \pm 0.04$ & $0.008 \pm 0.02$ & $0.007 \pm 0.03$ \\
\multirow{2}{*}{6} & branquias & $39.4 \pm 2.28$ & $46.5 \pm 2.22$ & $0.021 \pm 0.07$ & $14.9 \pm 1.88$ \\
& hígado & $33.7 \pm 1.19$ & $67.7 \pm 2.11$ & $0.007 \pm 0.02$ & $0.008 \pm 0.02$ \\
& cerebro & $0.015 \pm 0.04$ & $0.019 \pm 0.02$ & $0.007 \pm 0.02$ & $0.008 \pm 0.02$ \\
\hline
\end{tabular}

\section{Comparación de medias}

- Intervalos de confianza del $95.0 \%$ para la media de Est 1: $17.0657+/-15.162$ [1.90366, 32.2277]

- Intervalos de confianza del $95.0 \%$ para la media de Est 6: $16.8571+/-15.1398$ [1.71727, 31.9969]

- Intervalos de confianza del $95.0 \%$ intervalo de confianza para la diferencia de medias suponiendo varianzas iguales: $0.208583+/-20.1892$ [-19.9806, 20.3978]

\section{Prueba t para comparar medias}

- Hipótesis nula: media $1=$ media2

- Hipótesis Alt.: media1 <> media2

Suponiendo varianzas iguales: $\mathrm{t}=$ 0.0214261 valor- $\mathrm{P}=\mathbf{0 . 9 8 3 0 9 9}$

No se rechaza la hipótesis nula para alfa $=0.05$.
Puesto que el intervalo contiene el valor de 0 , no hay diferencia significativa entre las medias de las dos muestras de datos, con un nivel de confianza del 95.0\%. En este caso, la prueba se ha construido para determinar si la diferencia entre las dos medias es igual a 0.0 versus la hipótesis alterna de que la diferencia no es igual a 0.0 . Puesto que el valor-P calculado no es menor que 0.05 , no se puede rechazar la hipótesis nula.

Los resultados arrojados, asumen que las varianzas de las dos muestras son iguales. En este caso, esa suposición parece razonable con base en los resultados de la prueba-F para comparar las desviaciones estándar.

\section{Discusión}

La determinación de los parámetros físico-químicos arrojaron que, en general, todos se encontraron fuera del valor o rango establecido por la norma ambiental reguladora, por cuanto indicaron que las condiciones de calidad ambiental no son adecuadas. 
La demanda biológica de oxígeno, la demanda química de oxígeno y el oxígeno disuelto, justificaron más del $85 \%$ de la variabilidad dentro del sistema, por lo que puede mencionarse que se está consumiendo altas concentraciones de oxígeno para degradar la materia orgánica por parte de organismos aerobios, así como para oxidar químicamente la materia orgánica presente.

Resulta importante mencionar que en condiciones naturales, las aguas superficiales han sido por excelencia, el reservorio de destino final donde se descargan los residuales antropogénicos, afectando directamente el confort, alimentación, las reacciones frente estímulos y la propia defensa contra agentes patógenos naturales entre otras variables ambientales de interés; y sin duda alguna, esta situación representa un desequilibrio y afectación para el bienestar animal. Aunque el término de bienestar animal ha sido empleado solo para la experimentación a nivel de laboratorio, según Odendaal (2005), refiere a las condiciones que deben crearse para mantener niveles óptimos de salud y productividad. Sin embargo, una de las mayores interrogantes, en la actualidad, es conocer las posibles herramientas que puedan ser aplicadas para evaluar la salud o bienestar animal en su hábitat natural.

En el campo de la ecotoxicología, los cambios biológicos expresados por organismos, poblaciones o comunidades servirían como señales de la posible alteración que está sufriendo un ecosistema por las actividades de origen antropogénico. Cada nivel de respuesta biológica, representa una señal integrada de los niveles de contaminación en un área determinada y de esta manera, sirve como indicador del riesgo toxicológico a que una población natural está siendo expuesta (Orrego et al., 2005).

A nivel de ecosistemas acuáticos, los peces han sido uno de los primeros en ser utilizados en los protocolos de evaluación y como especies centinelas, ya que la característica más importante, es que están en la cumbre de la cadena trófica pudiendo afectar la salud humana, lo cual aumenta su importancia en los estudios ambientales (Zhou et al., 2008). En el caso del pez Gambusia punctata, es una especie de la familia Poeciliidae que habita de forma natural en los ríos cubanos donde además de su control biológico antivectorial de larvas, ha indicado la exposición ambiental de elementos tóxicos como son los metales pesados en las aguas (G. Argota, González, Argota, Fimia \& Iannacone, 2012).

En todas las estaciones, las concentraciones de la especiación de metales fueron similares, presentando características biodisponibles, por cuanto pudieron ser determinadas de forma bioacumulada tanto en branquias, hígado como cerebro.

Según Alquezar, Markich y Booth (2005), las mayores concentraciones en las branquias se justifica porque son los órganos que permiten la entrada, al igual que el oxígeno, de elementos esenciales ( $\mathrm{Cu}$ y $\mathrm{Zn}$ ) desde la columna de agua, la cual representa la matriz ambiental de mayor presencia de los mismos. De forma similar ocurre el ingreso permanente de otros elementos como $\mathrm{Pb}$ y $\mathrm{Cd}$, que al no tener una función biológica definida, pueden ser retenidos por las branquias, siendo los blancos iniciales de toxicidad por exposición a metales.

Una vez que el $\mathrm{Cu}$ y $\mathrm{Zn}$ son absorbidos por las branquias, se transportan al torrente sanguíneo hasta llegar al hígado, ya que el $\mathrm{Cu}$ interviene en el metabolismo del tejido conectivo, desarrollo óseo y función nerviosa, mientras que el $\mathrm{Zn}$ juega un papel importante en la regulación de la homeostasia (Widianarko, Van Gestel, Verweij \& Van Straalen, 2000), lo cual justifica la presencia de ambos elementos en este órgano, no así para $\mathrm{Pb}$ y $\mathrm{Cd}$. 
Finalmente, se concluyó que las aguas del ecosistema San Juan mostraron condiciones físico-químicas para la disponibilidad de metales, siendo corroborados mediante la bioacumulación en los tejidos analizados, por cuanto existió riesgo ecotoxicológico para la especie que se desarrolla en un ambiente natural.

\section{Referencias}

Albert, L. A. (1997). Evaluación de riesgo. En autor, Introducción a la toxicología ambiental (pp. 387). Metepec, México: Centro Panamericano de Ecología Humana y Salud. División de salud y ambiente.

Alquezar, R., Markich, S. \& Booth, D. J. (2005). Metal accumulation in the smooth toadfish, Tetractenos glaber, in estuaries around Sydney, Australia. Environmental Pollution, 142(1), 123131.

Argota, G., González, Y., Argota, H., Fimia, R. \& Iannacone, J. (2013). Desarrollo y bioacumulación de metales pesados en Gambusia punctata (Poeciliidae) ante los efectos de la contaminación acuática. Revista electrónica de veterinaria, 14(6), 1-13.

Carpenter, R. A. (1995). Risk assessment. Impact Assessment, 13, 153-187.

Van der Oost, R., Beyer, J. \& Vermeulen, N. P. (February, 2003). Fish bioaccumulation and biomarkers in environmental risk assessment: a review. Environmental Toxicology and Pharmacology, 13(2), 57-149.

Management Programme for the Gorgon Development: MPGD. (2004). Risk assessment process. In autor, Environmental Impact Statement/Environmental Review and Management Programme for the Gorgon Development (pp. 288-301).

Mongomery D. C. (1991). Diseño y Análisis de Experimentos. México D.F.: Grupo Editorial Iberoamérica S.A.

Odendaal, J. S. (August, 2005). Science-based assessment of animal wel- fare: companion animals. Revue scientifique et technique, 24(2), 493-502.

Orrego, R., Moraga-Cid, G., González, M., Barra, R., Valenzuela, A., Burgos, A. \& Gavilán J. F. (August, 2005). Reproductive, physiological, and biochemical responses in juvenile female Rainbow Trout (Oncorhynchus mykiss) exposed to sediment from pulp and paper mill industrial discharge areas. Environmental Toxicology and Chemistry, 24(8), 1935-1943.

Paustenbach, D. J. (1990). Health risk assessiment and the practice of indusrial hygiene. Am Ind Hyg Assoc J, 52, 339351.

Páez-Osuna, F. \& Osuna-Martínez, C. (2011). Biomonitores de la contaminación costera con referencia a las costas mexicanas: una revisión sobre los organismos utilizados. Hidrobiológica, 21(3), 229-238.

Plant, J., Smith, D., Smith, B. \& Williams, L. (2001). Environmental Geochemistry at the Global Scale. Applied Geochemistry, 16(1), 1291-1308.

Rainbow, P. S. (December, 1995). Biomonitoring of Heavy Metal Availability in the Marine Environment. Marine Pollution Bulletin, 31(4-12), 183-192.

Sigg, L., Black, F., Buffle, J., Cao, J., Cleven, R. \& Davidson, W. (2006). Comparison of Analytical Techniques for Dynamic Trace Metal Speciation in Natural Freshwaters. Environmental Science \& Technology, 40(6), 1934-1941.

Silveira, C. L. \& Oliveira-filho, E.C. (2013). Principios de toxicología Ambiental. Río de Janeiro, Brasil: Interciência. 
Statgraphics Plus (Versión 5.1.) [Software de computación]. Virginia, Estados Unidos: Statpoint Technologies, Inc.

Tercier-Waeber, M. L. \& Taillefert, M. (January, 2008). Remote in situ voltametric techniques to characterize the biogeochemical cycling of trace metals in aquatic systems. Journal of Environmental Monitoring, 10(1), 30-50.

U.S. Environmental Protection Agency: USEPA. (2000). Guidance for assessing chemical contaminant data for use in fish advisories. Fish sampling and analysis: Third Edition (Vol. 1). Washington, D.C.

West, D. W., Ling, N., Hicks, B. J., Tremblay, L. A., Kim, N. D. \& Van den Heuvel, M. R. (July, 2006). Cumula- tive impacts assessment along a large river, using brown bullhead catfish (Ameiurus nebulosos) populations. Environmental Toxicology and Chemistry, 25(7), 1868-1880.

Widianarko B., Van Gestel, C. A., Verweij, R. A. \& Van Straalen, N. M. (May, 2000). Associations between trace metals in sediment, water, and guppy, Poecilia reticulata (Peters), from urban streams of Semarang, Indonesia. Ecotoxicology and Environmental Safety, 46(1), 101-107.

Zhou, Q., Zhang, J., Fu, J., Shi, J. \& Jiang, G. (January, 2008). Biomonitoring: an Appealing Tool for Assessment of Metal Pollution in the Aquatic Ecosystem. Analytica Chimica Acta, 606(2), 135-150. 